\title{
ESTIMATIVA DOS PARÂMETROS CINÉTICOS DA PIRÓLISE DA MICROALGA Chlamydomonas reinhardtii
}

\author{
G. R. NUNES, L. A. ANDRADE, M. A. S. BARROZO, L. G. M. VIEIRA \\ Universidade Federal de Uberlândia, Faculdade de Engenharia Química \\ E-mail para contato: giovannarnunes@ hotmail.com
}

\begin{abstract}
RESUMO - Os combustíveis derivados de algas são uma alternativa promissora que tem sido estudada para suprir a demanda energética global. Neste trabalho, a microalga Chlamydomonas reinhardtii foi caracterizada utilizando diversas técnicas com a finalidade de determinar aspectos importantes de sua termoconversão, avaliando assim seu potencial para geração de biocombustíveis. $\mathrm{Na}$ análise elementar, observou-se que a biomassa estudada apresentou um alto teor de carbono e hidrogênio, em comparação ao oxigênio, o que pode ser um bom indicativo para formação de um bio-óleo de melhor qualidade. Os termogramas obtidos via análise termogravimétrica possibilitaram uma melhor compreensão da pirólise lenta da microalga Chlamydomonas reinhardtii. Por fim, o modelo de reações paralelas independentes utilizado no cálculo da energia de ativação mostrou-se adequado para descrever a pirólise lenta da microalga Chlamydomonas reinhardtii com coeficientes de correlação superiores a 0,966.
\end{abstract}

\section{INTRODUÇÃO}

A utilização de microalgas em processos termoquímicos vem ganhando cada vez mais visibilidade devido a sua capacidade de ultrapassar algumas das limitações advindas do uso de matérias-primas alimentares e resíduos lignocelulósicos, como a sazonalidade da cultura utilizada, dificuldades no transporte e armazenamento e uso de grande quantidade de terras férteis para seu cultivo (Bui et al., 2016). Nesse cenário, a microalga Chlamydomonas reinhardtii destaca-se por sua rápida reprodução e alta adaptabilidade a diversos ambientes.

O conhecimento dos parâmetros cinéticos da pirólise faz-se importante no projeto de reatores e gaseificadores; porém, este processo é complexo, sendo difícil propor mecanismos para caracterizar precisamente a decomposição da biomassa (Andrade et al., 2016). Dessa forma, além de caracterizar a biomassa visando a avaliar seu potencial para geração de bioóleo, um dos objetivos desse trabalho foi calcular os parâmetros cinéticos da reação de pirólise da microalga Chlamydomonas reinhardtii usando o modelo de reações paralelas independentes (RPI).

Este modelo é uma boa maneira de descrever a curva de termogravimetria derivada (DTG). Neste modelo, cada subcomponente é degradado individualmente, na mesma faixa de temperatura, garantindo a possibilidade de decomposições simultâneas. A taxa de perda de massa é calculada considerando a taxa das reações individualmente e suas respectivas frações. Diversos autores demonstraram em seus estudos que este modelo fornece parâmetros 
consistentes e com poucos erros, sendo conhecido por sua capacidade de descrever as informações de DTG e os estágios finais de decomposição da biomassa (Santos et al., 2010).

\section{METODOLOGIA}

\subsection{Meio de Cultivo da Microalga em Escala de Bancada}

A alga Chlamydomonas reinhardtii $\mathrm{CC}-124$ foi mantida em meio de Tri Acetato de Fosfato, com pH inicial de 7.

\subsection{Composição Química}

A composição protéica foi estimada pelo método Kjeldehl, enquanto as composições de lipídeos e carboidratos foram determinadas pelo método Soxhelt e método ácido fenolsulfúrico, respectivamente (Peng et al., 2001).

\subsection{Análise Termogravimétrica}

A perda de massa em função da temperatura foi conduzida em um analisador termogravimétrico da marca Shimadzu DTG 60, com razões de aquecimento de 5, 10, 20 e $30^{\circ} \mathrm{C} / \mathrm{min}$., nitrogênio como atmosfera inerte e faixa de temperatura de 30 a $900^{\circ} \mathrm{C}$.

\subsection{Análise Elementar, Imediata e Estimativa do Poder Calorífico Superior}

Os teores de carbono, hidrogênio e nitrogênio foram determinados utilizando um equipamento CHNS/O 2400 Perkin Elmer.

O teor de umidade, cinzas, voláteis e carbono fixo foi determinado pelas normas ASTM E871-82, ASTM E1534-93, ASTM E872-8 e ASTM Standard D3172, respectivamente.

O poder calorífico superior da biomassa foi estimado a partir da análise elementar, segundo a equação proposta por Protásio et al. (2011).

\subsection{Determinação dos Parâmetros Cinéticos}

Para determinação dos parâmetros cinéticos utilizou-se o modelo de reações paralelas independentes (RPI). Este modelo é conhecido por descrever de maneira satisfatória as informações de termogravimetria, pois considera interações entre carboidratos, proteínas e lipídeos na degradação da biomassa. As microalgas são principalmente compostas por proteínas, holoceluloses (carboidratos particularmente presentes na parede celular, como hemiceluloses e celulose), lipídeos e outros componentes, como pigmentos fotossintéticos (clorofilas, carotenóides e ficobilinas) (Bui et al., 2016). Neste estudo, foram assumidos cinco pseudo componentes para a pirólise das microalgas Chlamydomonas reinhardtii.

A taxa de conversão de cada subcomponente pode ser escrita individualmente, de acordo com a Equação 1: 


$$
\frac{d\left(X_{i}\right)}{d t}=k_{0 i} \exp \left(\frac{-E_{a i}}{R T}\right)\left(1-X_{i}\right)^{n_{i}}
$$

A perda de massa através do tempo pode ser calculada como indica a Equação 2:

$$
\frac{d m^{\text {calc }}}{d t}=-\left(m-m_{0}\right) \sum_{i=1}^{5} c_{i} \frac{d\left(X_{i}\right)}{d t}
$$

Os parâmetros $k_{0 i}, E_{a i}, n_{i}$ e $c_{i}$ foram estimados utilizando a técnica de regressão nãolinear, baseados nas informações da Termogravimetria Derivada (DTG), cuja função objetiva (F.O.) está descrita pela Equação 3:

$$
\text { F.O. } \left.{ }_{D T G}=\sum_{j=1}^{N}\left[\left(\frac{d m(t)_{j}}{d t}\right)^{o b s}-\frac{d m(t)_{j}}{d t}\right)^{c a l c}\right]^{2}
$$

Para a estimativa dos parâmetros cinéticos, foi utilizado um programa baseado no método de evolução diferencial (Santos et al., 2010). Por sua vez, a otimização foi realizada utilizando o software Matlab.

\section{RESULTADOS E DISCUSSÕES}

\subsection{Composição Química}

Através da análise da composição química, obteve-se $61,73 \%$ de proteínas, 3,28\% de carboidratos e $12,19 \%$ de lipídeos. Nota-se que há elevado teor proteico, o que, em biomassas, de forma geral, indica alto poder calorifico (Boateng et al., 2010).

\subsection{Análise Termogravimétrica}

Na Figura 1 são mostrados os termogramas determinados para a microalga analisada.

Figura 1- Termogramas da microalga Chlamydomonas sp.
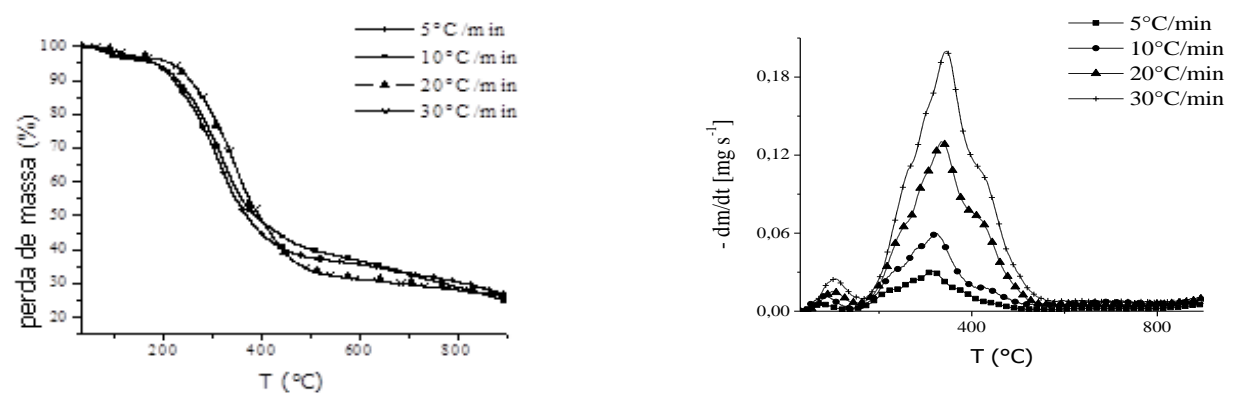
Os termogramas apontam três etapas principais na decomposição de microalgas. Na primeira etapa, a remoção do teor de umidade, que ocorre entre $30^{\circ} \mathrm{C}$ e $150^{\circ} \mathrm{C}$, e a quebra de uma parte dos lipídeos que se finaliza a $190^{\circ} \mathrm{C}$ (Marcilla et al., 2013). Além disso, durante a faixa de temperatura de $80^{\circ} \mathrm{C}$ a $120^{\circ} \mathrm{C}$, alguns pigmentos fotossintéticos ainda podem se degradar, como as clorofilas A e B (Weemaes et al., 1999). A segunda etapa consiste principalmente na decomposição de carboidratos (hemiceluloses, celulose) e proteína $\left(190^{\circ} \mathrm{C}\right.$ $430^{\circ} \mathrm{C}$ ). Esta etapa concentra a principal perda de massa de todo o processo, que foi de aproximadamente $63 \%$. A terceira etapa $\left(430^{\circ} \mathrm{C}-750^{\circ} \mathrm{C}\right)$ mostrou uma degradação de lipídeos, especialmente aqueles associados com as cadeias de ácidos graxos (Figueira et al., 2015).

\subsection{Análise Elementar, Imediata e Poder Calorífico}

Pela análise elementar, obteve-se 41,23\% de carbono, 7,21\% de hidrogênio, 8,81\% de nitrogênio, $1,45 \%$ de enxofre e $41,3 \%$ de oxigênio. A biomassa estudada apresentou alto teor de carbono o que pode ser um indicativo para formação de bio-óleo de melhor qualidade. Para a análise imediata, encontrou-se $70,83 \%$ de material volátil, $11,21 \%$ de carbono fixo e $17,96 \%$ de cinzas. O poder calorífico superior estimado foi de $20,47 \mathrm{MJ} / \mathrm{kg}$, superior a diversos resíduos lignocelulósicos, como o tegumento de manga, que foi de $18,54 \mathrm{MJ} / \mathrm{kg}$ (Andrade et al., 2016).

\subsection{Determinação dos Parâmetros Cinéticos}

Na Tabela 1 são apresentados os parâmetros cinéticos estimados pelo modelo RPI.

Tabela 1 - Parâmetros cinéticos da pirólise da microalga pelo modelo RPI

\begin{tabular}{|c|c|c|c|c|c|c|c|}
\hline B & Reação & Ordem & $\begin{array}{c}\text { Composição } \\
\text { (\% massa) }\end{array}$ & $k_{0, I}\left(\mathrm{~s}^{-1}\right)$ & $\boldsymbol{E}_{a, i}$ & $\mathbf{R}^{2}$ & Desvio \\
\hline \multirow{5}{*}{5} & $\mathrm{R}_{1}$ & 1,28 & 0,04 & $5,04 \cdot 10^{+11}$ & 93 & \multirow{5}{*}{0,966} & \multirow{5}{*}{5.3} \\
\hline & $\mathrm{R}_{2}$ & 1,17 & 0,09 & $2,29.10^{+10}$ & 119 & & \\
\hline & $\mathrm{R}_{3}$ & 1,33 & 0,16 & $9,48.10^{+08}$ & 116 & & \\
\hline & $\mathrm{R}_{4}$ & 1,67 & 0,34 & $3,15 \cdot 10^{+08}$ & 123 & & \\
\hline & $\mathrm{R}_{5}$ & 1,77 & 0,27 & $9,87.10^{+07}$ & 130 & & \\
\hline \multirow{5}{*}{10} & $\mathrm{R}_{1}$ & 1,23 & 0,04 & $4,55.10^{+11}$ & 96 & \multirow{5}{*}{0,971} & \multirow{5}{*}{4,7} \\
\hline & $\mathrm{R}_{2}$ & 1,27 & 0,09 & $1,1210^{+10}$ & 116 & & \\
\hline & $\mathrm{R}_{3}$ & 1,28 & 0,15 & $9,44.10^{+08}$ & 117 & & \\
\hline & $\mathrm{R}_{4}$ & 1,67 & 0,32 & $2,99.10^{+08}$ & 123 & & \\
\hline & $\mathrm{R}_{5}$ & 1,77 & 0,16 & $6,41 \cdot 10^{+07}$ & 133 & & \\
\hline \multirow{5}{*}{20} & $\mathrm{R}_{1}$ & 1,23 & 0,03 & $2,67 \cdot 10^{+11}$ & 99 & \multirow{5}{*}{0,984} & \multirow{5}{*}{3,5} \\
\hline & $\mathrm{R}_{2}$ & 1,27 & 0,09 & $2,07.10^{+10}$ & 119 & & \\
\hline & $\mathrm{R}_{3}$ & 1,28 & 0,15 & $9,48.10^{+08}$ & 117 & & \\
\hline & $\mathrm{R}_{4}$ & 1,67 & 0,34 & $3,00.10^{+08}$ & 122 & & \\
\hline & $\mathrm{R}_{5}$ & 1,77 & 0,27 & $7,48 \cdot 10^{+07}$ & 130 & & \\
\hline \multirow{5}{*}{30} & $\mathrm{R}_{1}$ & 1,23 & 0,03 & $2,95 \cdot 10^{+11}$ & 99 & \multirow{5}{*}{0,987} & \multirow{5}{*}{3,0} \\
\hline & $\mathrm{R}_{2}$ & 1,33 & 0,09 & $2,07.10^{+10}$ & 120 & & \\
\hline & $\mathrm{R}_{3}$ & 1,28 & 0,15 & $8,58.10^{+08}$ & 116 & & \\
\hline & $\mathrm{R}_{4}$ & 1,66 & 0,34 & $3,00 \cdot 10^{+08}$ & 121 & & \\
\hline & $\mathrm{R}_{5}$ & 1,77 & 0,27 & $8,26 \cdot 10^{+07}$ & 129 & & \\
\hline
\end{tabular}


$\mathrm{Na}$ Tabela anterior, $\mathrm{R}_{1}$ corresponde a degradação dos pigmentos fotossintéticos e lipídeos, $\mathrm{R}_{2}$ às hemiceluloses, $\mathrm{R}_{3}$ às celuloses, $\mathrm{R}_{4}$ às proteínas e $\mathrm{R}_{5}$ aos lipídeos e materiais que não volatizaram em estágios anteriores de decomposição, assim como outros componentes da biomassa. As informações experimentais indicaram que a taxa de aquecimento não afetou significantemente a estimativa da energia de ativação. A faixa de valores de energia de ativação encontrada para $\mathrm{R}_{1}$ coincide com o trabalho de Figueira et al. (2016) (91-98 kJ/mol) para a Chlorella vulgaris. Para $\mathrm{R}_{2}$, o valor estimado também coincidiu com valores na literatura (90-125 kJ/mol) (Bui et al., 2016; Andrade et al., 2016). Entretanto, na reação $\mathrm{R}_{3}$, os valores da energia de ativação $(116-117 \mathrm{~kJ} / \mathrm{mol})$ foram mais baixos que os encontrados na literatura (Bui et al., 2016). Isto aconteceu, pois, este estudo, diferentemente de outros reportados anteriormente, considerou uma ampla faixa de temperaturas e também incluiu componentes adicionais, o que promoveu melhor descrição das informações experimentais pelo modelo. Para $\mathrm{R}_{4}$, os valores de energia de ativação foram próximos aos reportados na literatura (Figueira et al., 2015). Finalmente, $\mathrm{R}_{5}$ apresentou os valores mais altos. Diferentes valores de energia de ativação foram encontrados para lipídeos ( $\mathrm{R}_{5}$ ), como Bui et al. (2016) que constatou $114 \mathrm{~kJ} / \mathrm{mol}$ e $134 \mathrm{~kJ} / \mathrm{mol}$ utilizando Chlamydomonas sp e Chlorella sorokiniana, respectivamente. Isto ocorre, pois, este componente tem uma degradação térmica complexa quando comparado aos outros componentes da biomassa.

Na Figura 2 são mostrados os resultados previstos pelo modelo RPI e as medidas experimentais para a taxa de $20^{\circ} \mathrm{C} \% \mathrm{~min}$.

Figura 2 - Curvas experimentais e calculadas pelo DTG pelo modelo RPI a $20^{\circ} \mathrm{C} / \mathrm{min}$.

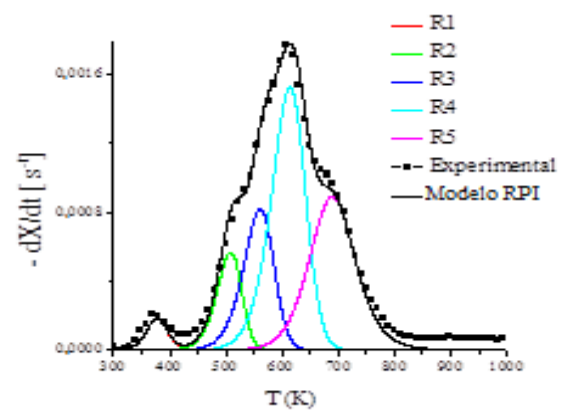

Observou-se que modelo RPI forneceu uma boa representação da pirólise da microalga estudada, especialmente para taxas de aquecimento elevadas.

\section{CONCLUSÃO}

O trabalho possibilitou melhor conhecimento sobre a microalga Chlamydomonas reinhardtii e seu comportamento durante a pirólise. A análise elementar foi um bom indicativo para a formação de bio-óleo de melhor qualidade ao comparar os teores de carbono e hidrogênio com o oxigênio. O modelo de reações paralelas independentes utilizado mostrou-se adequado para descrever a cinética de degradação. 


\section{NOMENCLATURA}

Calc: dados calculados pelo modelo RPI (-)

$E_{\text {ai: }}$ energia de ativação $(\mathrm{kJ} / \mathrm{mol})$

$\mathrm{k}_{0 \mathrm{i}}$ : fator pré exponencial (a depender de $\mathrm{n}_{\mathrm{i}}$ )

N: número de dados experimentais para cada série (-)

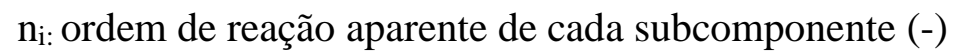

Obs: dados observados experimentalmente (-)

$\mathrm{X}_{\mathrm{i}}$,: conversão (-)

\section{REFERÊNCIAS}

ANDRADE, L.A.; BARROZO, M. A. S.; VIEIRA, L. G. M. Thermo-chemical behavior and product formation during pyrolysis of mango seed shell. Ind. Crops Prod. 85, 174-180, 2016.

BOATENG, A.A.; MUlLEN, C. A.; GOLDBERG, N. M., 2010. Producing Stable Pyrolysis Liquids from the Oil Seed Presscakes of Mustard Family Plants: Pennycress (Thlaspiarvense L.) and Camelina (Camelina sativa)†, Energy \& Fuels, 24, 66246632 , 2010.

BUI, H. H.; TRAN, K. Q; CHEN, W. H. Pyrolysis of microalgae residues - A kinetic study. Ioresource Technology, 362-366, 2016

CHEN, C.; MA, X.; HE, Y. Co-pyrolysis characteristics of microalgae Chlorella vulgaris and coal through TGA. Bioresource Technology 117, 264-273, 2011.

FIGUEIRA, C. E.; MOREIRA, P.F. Jr.; GIUDICI, R. Thermogravimetric Analysis of the Gasification of Microalgae Chlorella vulgaris, Bioresour. Technol. 198, 717-724, 2015.

MARCILLA, A.; CATALÁ, L.; GARCÍA-QUESADA, J. C.; VALDÉS, F. J.; HERNÁNDEZ, M. R. A review of thermochemical conversion of microalgae. Renew. Sustain. Energy Rev. 27, 11-19, 2013.

PENG, W.; WU, Q.; TU, P. Pyrolytic characteristics of heterotrophic Chlorella protothecoides for renewable bio-fuel production. J. Appl. Phycol. 13 (1), 5-12, 2001.

PROTÁSIO, T. P.; BUFALINO, L.; TONOLI, G. H. D.; COUTO, A. M.; TRUGILHO, P. F.; GUIMARÃES JÚNIOR, M. Relação entre o poder calorífico superior e os componentes elementares e minerais da biomassa vegetal. Pesquisa Florestal Brasileira, Colombo, v. 31, n. 66, p. 122-133, 2011.

SANTOS, K. G.; LIRA, T. S.; MURATA, V. V.; GIANESELLA, M.; BARROZO, M. A.S. "Pyrolysis of Sugarcane Bagasse: A Consecutive Reactions Kinetic Model from TGA Experiments", Materials Science Forum, Vols. 660-661, pp. 593-598, 2010

WEEMAES, C. A.; VEERLEOOMS, A. M.; LOEY, V.; HENDRICKX, M. E. Kinetics of Chlorophyll Degradation and Color Loss in Heated Broccoli Juice. J. Agric. Food Chem. 47, 2404-2409, 1999. 